\title{
Belphégor
}

\section{Fantastique Charles Grivel}

\section{Marc Lits}

\section{(2) OpenEdition}

\section{Journals}

Electronic version

URL: https://journals.openedition.org/belphegor/1354

DOI: 10.4000/belphegor.1354

ISSN: 1499-7185

\section{Publisher}

LPCM

\section{Electronic reference}

Marc Lits, "Fantastique Charles Grivel", Belphégor [Online], 16-1 | 2018, Online since 04 July 2018, connection on 19 October 2021. URL: http://journals.openedition.org/belphegor/1354 ; DOI: https:// doi.org/10.4000/belphegor.1354

This text was automatically generated on 19 October 2021.

\section{(c) (i) (9)}

Belphégor est mis à disposition selon les termes de la Licence Creative Commons Attribution - Pas d'Utilisation Commerciale - Pas de Modification 4.0 International. 


\title{
Fantastique Charles Grivel
}

\author{
Marc Lits
}

En 1992, Charles Grivel publiait son essai Fantastique-Fiction ${ }^{1}$, texte qui se situait dans la droite ligne des préoccupations qui étaient les siennes depuis sa thèse consacrée à « la production de l'intérêt romanesque ", à savoir un intérêt marqué pour les productions de masse, et non les seuls auteurs légitimes, mais aussi pour les images, indissociables des textes. Sa thèse, publiée chez Mouton en 1973, sera longtemps dénigrée (mais pas par tous, comme en témoigne la critique de Jacques Dubois publiée dans la revue Littérature en $1974^{2}$ ), mais il y pose les bases de toutes les analyses ultérieures qui seront entreprises par un chercheur sans cesse décalé, jusqu'à la provocation, qui partait du principe que c'est en analysant les marges, les périphéries qu'on comprend le mieux le fonctionnement général du système.

Charles Grivel ne pouvait pas ignorer le genre fantastique, dans la mesure où, plus que d'autres, c'est un système narratif qui fonctionne par production d'images, qui repose sur la fascination pour des images dérangeantes, comme l'a montré Freud, en déclinant la notion d'Unheimlich dans sa lecture de L'homme au sable de E.T.A. Hoffmann ${ }^{3}$. Cet intérêt pour l'image, sous toutes ses formes, la gravure, les illustrations des romans du XIXe siècle qu'il chérissait particulièrement, la bande dessinée, la photographie, le cinéma, il peut aussi le rencontrer dans les grands textes des fantastiqueurs, jusqu'à ce Dracula dont il se fait le lecteur passionné pour le numéro des Cahiers de l'Herne qu'il dirigea en 1997. Ainsi, entre les recherches sur l'image qu'il développe autour de la revue MANA qu'il contribua à créer à Mannheim et les travaux qu'il consacre au genre fantastique, il y a une continuité évidente.

Quand Charles Grivel commence à s'intéresser à ce genre déconsidéré (ce qui ne peut, pour lui, qu'ajouter à sa séduction), les études sur le fantastique sont encore rares en France, et plutôt consacrées à une histoire ou à une thématique du genre, dans la lignée des travaux fondateurs de Pierre-Georges Castex ou de Roger Caillois. Mais le structuralisme passa par là et Tzvetan Todorov commit un essai qui aura un impact considérable, Introduction à la littérature fantastique ${ }^{4}$. Le premier mot du titre, dans sa modestie affichée, révèle en fait qu'il s'agit surtout d'un exercice de séminaire de recherche, où le fantastique, comme le policier un peu plus tard, est l'occasion de 
démontrer que les caractéristiques d'un genre ne doivent rien aux thèmes qu'il exploite, ou à un certain type de héros mis en scène, mais qu'elles s'observent dans la récurrence de structures narratives figées. Ces sous-genres (parce que leur répétitivité organisationnelle permet à la fois aux chercheurs de fonder leur modèle théorique d'analyse structurale mais aussi de justifier, dans la foulée, la pauvreté créatrice de récits dus à d'habiles faiseurs dénués du génie créateur qui fait les grandes œuvres originales) sont donc des productions absentes de toute littérarité, condamnées à se reproduire dans des codes figés. Même si l'étude de Todorov ne porte que sur quatre ou cinq romans, et peut facilement être réfutée aujourd'hui, tant elle monte en généralité au départ d'un corpus trop réduit, qui s'abstient délibérément de prendre en compte des œuvres plus variées, et qui réduit les textes analysés à de purs enchaînements séquentiels, elle sera cependant source de nombreuses lectures réductrices d'un genre aussi hétérogène que porteurs d'interrogations et de fascinations multiples pour d'innombrables lecteurs de par le monde.

Charles Grivel, dans sa thèse, s'inscrivait dans une tension permanente entre deux champs de l'analyse littéraire, qui commençaient à affirmer leur domination sur la recherche littéraire après le linguistic turn des années soixante, la sémiotique structurale et la sociocritique. Il aurait donc pu utiliser ces lunettes analytiques pour se confronter aux auteurs fantastiques, mais il eut l'intelligence et la finesse de s'en garder, pour tenter de saisir l'essence du fantastique, dans sa lecture très personnelle et dans un style qui essaye de rendre compte de l'étrangeté inquiétante de cet objet si singulier.

5 Au moment où son livre est sorti, je coordonnais un numéro d'une revue consacrée à la littérature française de Belgique, Textyles ${ }^{5}$, qui voulait mettre en avant quelques auteurs qui s'étaient illustrés dans un genre très prisé dans un pays à la frontière entre les mondes latin et germanique. Maurice Maeterlinck, Georges Rodenbach, Jean Ray, Michel de Ghelderode, Jean Muno, Gaston Compère y étaient analysés, comme Edgar P. Jacobs et même Hergé pour la bande dessinée. Au-delà de la préface classique pour ce type de dossier, dressant le panorama du genre en Belgique et tentant d'en dégager quelques spécificités, il nous avait semblé intéressant de demander un article à celui qui venait de publier un essai aussi décapant sur le genre que nous étudiions. Je ne connaissais pas encore Charles, que j'allais avoir le plaisir de rencontrer quelques années plus tard quand quelques amateurs de littérature populaire allaient se rassembler pour fonder ce qui deviendrait la LPCM, mais je lui écrivis pour solliciter de sa part un article présentant sa conception du genre fantastique, en lien direct avec son ouvrage.

6 Il me répondit, dans le style un peu bougon que ceux qui l'ont fréquenté reconnaîtront, qu'il n'avait rien à ajouter à son livre et qu'il ne voyait pas l'intérêt d'y adjoindre un article supplémentaire, puisque tout s'y trouvait. J'insistai, en lui proposant alors une forme de dialogue, en lui envoyant quelques questions auxquelles il pourrait répondre sans aucune contrainte, ni limite, ni contrôle. L'exercice lui convint, car l'on sait que Charles était un homme d'oralité, que ses communications à des colloques étaient toujours un exercice de style oral plus qu'écrit, faussement improvisé mais brillamment présenté.

7 Et ce n'est pas par hasard que cet échange épistolaire commence par la question du regard, de la vision, où il se démarque d'emblée des lectures structuralistes à la Genette, alors dominantes, pour insister sur la dimension visuelle du fantastique, à 
travers les textes et les imageries, les fantasmagories qu'ils font surgir. Il avance là sa conception personnelle du texte littéraire et de l'acte de lecture, démontrant qu'il ne fut pas proche de Hans Robert Jauss, comme lecteur à l'Université de Giessen, par hasard: "Ce n'est pas le savoir qui agit, mais l'œil ». Il met aussi en question les typologies génériques, commodes pour les chercheurs et les bibliothécaires, mais il préfère le terme plus large de "fiction", laquelle englobe bien sûr les littératures populaires dont il vante les « délices ». Et c'est en cela que ces sous-genres réprouvés, le fantastique comme le policier, sont pour lui étonnamment modernes, voire postmodernes. Entre autres parce ces textes font émerger le désir, placent le corps en leur centre, même quand il s'agit de corps morcelés, torturés, comme ils se retrouvent aussi chez Georges Bataille ou dans les tableaux de Max Ernst. Ce qui montre encore que, pour Charles Grivel, entre texte et image, entre littérature populaire et créateurs se revendiquant du surréalisme, il n'y a pas de frontière, mais des filiations, des expressions communes d'un désir à fleur de texte et d'image. "La légèreté est grave ", disait-il en conclusion, comme la vie, comme la mort. C'est ainsi qu'il lisait, qu'il écrivait, qu'il vivait.

Le hasard (mais nous savons qu'il ne s'agit pas de cela) veut que je revienne aujourd'hui à ce numéro de la revue Textyles pour rendre hommage à Charles Grivel, alors que je clôturais la préface du dossier de ce même numéro en rendant un autre hommage, à Jean Fabre ${ }^{6}$, qui avait publié lui aussi plusieurs études sur le fantastique et qui m'avait promis un article consacré à Monique Watteau, l'auteur de La colère végétale et de L'ange à fourrure. Il décéda quelques mois avant le bouclage du dossier et n'avait donc pu honorer son engagement. Décidément, le genre fantastique est bien un lieu où les vivants et les morts se donnent la main, Charles en aurait ri avec malice.

\section{NOTES}

1. Charles GRIVEL, Fantastique-Fiction, Paris, PUF, coll. "Ecriture", 1992.

2. Jacques DUBOIS, "Production de l'intérêt romanesque de Jacques Dubois", Littérature, 1974, vol. 16, $\mathrm{n}^{\circ} 4$, pp. 102-106.

3. Sigmund FREUD, L'inquiétant familier (suivi du Marchand de sable de E.T.A. Hoffmann), Paris, Payot, coll. "Petite Bibliothèque Payot", 2012.

4. Tzvetan TODOROV, Introduction à la littérature fantastique, Paris, Seuil, 1970.

5. Textyles, «Fantastiqueurs ", n 10, 1993. Disponible sur le site Revues.org.

6. Il venait de publier Le miroir de sorcière. Essai sur la littérature fantastique, Paris, Corti, 1992. 
INDEX

Mots-clés: Grivel, illustrations, sérialité, littérature populaire, culture médiatique, fantastique

\section{AUTHOR}

MARC LITS

Université catholique de Louvain 